\title{
Development of a Plasma Chemistry Model for Helicon Plasma Thruster analysis
}

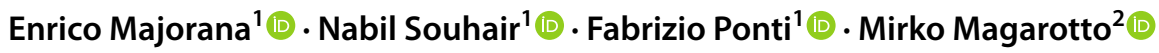

Received: 28 April 2021 / Revised: 11 July 2021 / Accepted: 22 July 2021 / Published online: 25 September 2021

(c) The Author(s) 2021

\begin{abstract}
The present work is part of a wider project aimed at improving the description of the plasma dynamics during the production phase of a Helicon Plasma Thruster. In particular, the work was focused on the development of a chemical model for Argon- and Xenon-based plasma. The developed model consists of a collisional radiative model suitable to describe the dynamics of the $1 s$ and $2 p$ excited levels. The model is meant to be complementary to 3D-VIRTUS, a numerical tool which enforces a fluid description of plasma, developed by the University of Padova to analyse helicon discharges. Once identified, the significant reactions for both propellants, the reaction rate coefficients, have been integrated exploiting cross sections from literature and assuming a Maxwellian velocity distribution function for all the species. These coefficients have been validated against experimental measurements of an Argon Inductively Coupled Plasma and compared with a well-established code. For Argon, the selected reactions have been reduced through a proposed lumping methodology. In this way, it was possible to reduce the number of equations of the system to solve, and implement them into 3D-VIRTUS. A validation against an experimental case taken from literature was performed, showing good agreement of the results. Regarding the Xenon model, only a verification has been performed against the results of another collisional-radiative model in literature. Finally, a predictive analysis of the propulsive performances of a Helicon Plasma Thruster for both Argon and Xenon is presented.
\end{abstract}

Keywords Collisional radiative model $\cdot$ Lumping methodology $\cdot$ Plasma density $\cdot$ Plasma thruster $\cdot$ Helicon discharges

\section{Introduction}

Argon and Xenon are two of the most common propellants in the electric propulsion segment $[1,2]$. Argon is widely used for plasma source operations, thanks to its availability and low cost. As a consequence, in literatures, many physical and chemical data are available, such as electronic impact

Enrico Majorana

enricomajorana@gmail.com

Nabil Souhair

nabil.souhair2@unibo.it

Fabrizio Ponti

fabrizio.ponti@unibo.it

Mirko Magarotto

mirko.magarotto@unipd.it

1 Dipartimento di Ingegneria Industriale, Alma Mater Studiorum-Università di Bologna, Via Fontanelle 40, 40121 Forlì, FC, Italy

2 Dipartimento di Ingegneria Industriale, Univeristà di Padova, Via Venezia 15, 35131 Padua, PD, Italy cross sections and radiative transition parameters. On the other hand, Xenon is usually preferred due to its good propulsive performance [1] and to its advantages, such as a low ionization potential and non-toxicity [3]. Both gases can be used as propellants in Helicon Plasma Thrusters (HPT).

Recently, the HPT has gained a great deal of interest among the space propulsion field $[4,5]$. The working principle of an HPT is essentially based on two stages: the plasma production and the acceleration phase, as shown in Fig. 1. During the production stage, the gas is injected into a dielectric tube, which is wrapped by a Radio Frequency (RF) excitation antenna and surrounded by coils or permanent magnets, that generate a magnetic field. The RF antenna deposits power into the electrons in the plasma source region, ionizing the injected neutral gas through propagation of helicon waves [6].

The magnetic field in the plasma source is assumed to be uniform and its task is to radially confine the plasma and to improve the propagation of the helicon waves in the production stage [7]. The acceleration stage is provided by the divergence of the magnetic field lines in the 


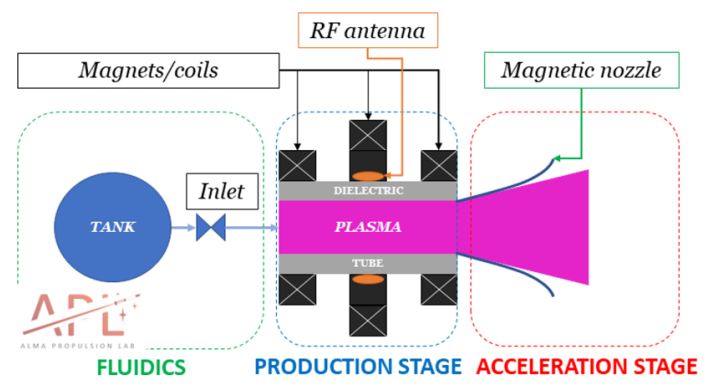

Fig. 1 Schematic of Helicon Plasma Thruster

plasma plume, the so-called magnetic nozzle effect [7, 8]. However, the magnetic field lines close themselves on the spacecraft structure, therefore, the determination of the exact conditions for the detachment becomes a critical issue to provide net momentum and is still under investigation [9]. Thanks to the absence of electrodes, the HPT avoids two life-time limiting processes that affect ionthrusters and hall thrusters [2], acceleration grid erosion and wall-cathode erosion, respectively. As a consequence, the HPT is a more reliable and cost-effective alternative for propelling small spacecrafts (MicroSat, CubeSat). Some examples of HPT with a high input power, above $1 \mathrm{~kW}$, are: the VASIMIR thruster [10], and the recent HPT05 [11] manufactured by SENER. A promising HPT for small spacecraft application is REGULUS [12,13], developed by T4i S.p.a [14] and CISAS-University of Padova [15]. The future step in the development process of REGULUS has started in March 2021 and consists of an in-orbit-demonstration (IOD) $[16,17]$ of the engine.

The development of a numerical tool is required to describe the physical phenomena happening within a HPT. In this regard, the simulated performances of an HPT depend strictly on the proper modelling of the plasma dynamics. The University of Padova, in cooperation with the University of Bologna, has developed a numerical code used to simulate the dynamics of the plasma inside a HPT, namely 3D-VIRTUS [18]. 3D-VIRTUS is a numerical tool based on two modules: the Electro Magnetic (EM) module that accounts for the deposition of the power provided by the antenna into the plasma by solving the plasma-wave interaction [19], and the fluid module which solves the plasma transport to determine the temperature and density distribution of the species considered. The decoupling of the two processes is allowed since the process of propagation and absorption of EM waves by the plasma has time scales in the order of $10^{-6}$. While, the plasma reconfigure itself under the forcing action of the RF deposited power with time scales in the order of $10^{-3}$ [18]. The fluid module is based on a system of balance equations for energy, momentum, and mass:

$\frac{\partial n_{k}}{\partial t}+\nabla \cdot \Gamma_{k}=R_{k}$

$\frac{\partial n_{\epsilon}}{\partial t}+\nabla \cdot \Gamma_{\varepsilon}+E_{\mathrm{DD}} \cdot \Gamma_{e}=R_{\varepsilon}$

$\nabla^{2} \phi=-q\left(\frac{n_{i}-n_{e}}{\varepsilon_{0}}\right)$

where Eq. (1) represents the continuity equation, with $\mathrm{k}$ ranging throughout each species considered. Equation (2) is the energy balance of the electron energy density $n_{\epsilon}$, from which the electron temperature $T_{e}$ can be derived considering $n_{\epsilon}=3 / 2 n_{e} T_{e}^{(v)}$. The electric field generated by the local imbalance of net charge is considered via the $E_{\mathrm{DD}}$ term, which is computed from the Poisson equation, Eq. (3). The $\Gamma_{k}$ and the $\Gamma_{\varepsilon}$ terms represent the flux of particles and the electron energy flux, respectively, and are derived from the drift diffusion assumption:

$\Gamma_{k}=n_{k} \mathbf{v}_{k}= \pm \overline{\bar{\mu}}_{k} n_{k} E_{\mathrm{DD}}-\overline{\bar{D}}_{k} \nabla n_{k}$

$\Gamma_{\varepsilon}=-\overline{\bar{\mu}}_{\varepsilon} n_{\varepsilon} E_{\mathrm{DD}}-\overline{\bar{D}}_{\varepsilon} \nabla n_{\varepsilon}$

where $v_{k}$ is the species velocity, $\overline{\overline{D_{k}}}$ is the species diffusivity following from Einstein's relation, and $\overline{\overline{\mu_{k}}}=|q| / n v$, is the species mobility, which is non-zero for charged species only, and has positive (negative) values for ions (electrons). In Eq. (5), $\overline{D_{\varepsilon}}$ and $\overline{\overline{\mu_{\varepsilon}}}$ represent the electron energy diffusivity and mobility, respectively. The heavy species are considered under the hypothesis of isothermal equilibrium that allows to decouple the energy equation for that species. Then, the contribution of the chemical phenomena (e.g. ionization of the gas propellant, excitation of neutral atoms and so on...) is given by the source/sink terms appearing inside these balance equations, namely $R_{k}, R_{\varepsilon}$.

In [18], numerical results of 3D-VIRTUS have been compared to experimental measurements obtained on a Piglet reactor [34], for Argon propellant. The calculated electron density showed the same trend of the experiment, yet with errors within one order of magnitude. The quantitative difference between the numerical and the experimental results has been attributed, even though in a minor role, also to the set of chemical reactions and species considered inside the code [18]. Thus, it was necessary to develop an improved chemical model to be implemented into 3D-VIRTUS, in order to describe the reactions taking place within the plasma source. 


\section{Collisional-Radiative Model}

The developed model is a collisional-radiative model (CRM), used to investigate the population density of the different excited states of Xenon and Argon atoms in a condition of weak ionization, low pressure and low temperature. A CRM describes the populating and depopulating mechanism of the species inside a plasma. In case of mono-atomic elements, the following groups can be considered: electrons, ions, neutral atoms, excited atoms. In particular, the excited atoms represent a large number of different species, and the description of their interacting mechanisms becomes fundamental in low-temperature plasma [20]. In general, CRMs consider two sets of interaction between species inside the plasma: collisional reactions and radiative decay of the excited states, from higher to lower levels of excitation. For low-pressure and low-temperature plasma, the two main processes to take into account are [21-24]:

- electronic impact

- optical radiative transition

Due to the low-pressure conditions of neutral atoms, tenths of mTorr for HPT applications, reactions between heavy particles, such as charge exchange resonant and non-resonant collisions (CEX) or 3-body recombination, have not been considered [20]. The populating and depopulating mechanisms considered are reported in Eq. (6), that express the population balance for each species:

$$
\begin{aligned}
\frac{\mathrm{d} n_{i}}{\mathrm{~d} t}= & \sum_{j \neq i} n_{e} n_{j} K_{j i}^{e x}+\sum_{j>i} n_{j} A_{j i} \Lambda_{j i} \\
& -\left(n_{i} \sum_{j \neq i} n_{e} K_{i j}^{e x}+n_{i} \sum_{j<i} A_{i j} \Lambda_{i j}+n_{e} n_{i} K_{i}^{i z}\right)
\end{aligned}
$$

then, the population density of excited level $n_{i}$ is now coupled with the population density of the excited level $n_{j}$. To obtain the population density of each excited state, the set of coupled differential equations has been numerically solved. On the right-hand side of the population balance, the first part represents the terms related to the production of $i$-th species, while the terms inside the brackets represent its destruction mechanisms. The reactions are due to de/excitation and spontaneous radiation from higher levels, while the ionization term is written separately as, $n_{e} n_{i} K_{i}^{i z}$. The electronic impact reactions are modelled with the reaction rates $K_{i j}^{\mathrm{ex}}, K_{j i}^{\mathrm{ex}}$, responsible for excitation and de-excitation process, and the reaction rate $K_{i}^{i z}$ used for the ionization of the state. The spontaneous radiation is taken into account for transition from i-th to j-th level with two coefficients: $A_{i j}$ and $\Lambda_{i j}$, respectively, the transition probability (or Einstein's coefficient) and the escape factor.
In the developed model, only the kinetic mechanisms among $1 s$ and $2 p$ excited levels (Paschen notation) have been considered, since the typical working pressure of the HPT is within the range for excitation of mostly these levels [20]. In particular, the transitions mechanisms of $1 s$ and $2 p$ to higher excited levels have been neglected. The excited levels considered establish, including the ion and the ground state, a total of 16 species. In "Appendix", all the excitation and de-excitation reactions considered for the Argon and Xenon model are reported, together with the reactions due to elastic scattering, ionization and radiative transition. In this latter case, the reactions are specified with the reference of the cross section used or if available the analytical rate coefficient.

In Tables 1 and 2, the selected levels for Argon and Xenon are reported with their energy potential in the Racah and Paschen notation. The physical data, needed for modelling the CRM, such as statistical weights, energy levels, Einstein coefficients, have been recovered from the NIST database [25].

\subsection{Collisional Reactions}

The key element of the population model is the rate coefficient $K_{i j}^{\text {ex }}$, that represents how many species are created/ destroyed in a time interval by electronic impact. In general, the rate coefficient, which depends mainly on electron temperature, can be computed directly by integrating the electron energy distribution function (EEDF) with the cross section of the selected reaction [22, 23, 27]. In the developed model, some empirical cross sections for each

Table 1 Paschen and Racah notation of state levels of Ar I [24]

\begin{tabular}{lll}
\hline Paschen notation & Argon (Ar I) & \\
\cline { 2 - 3 } & Racah notation & Energy \\
\hline$g s$ & $3 p^{6}$ & 0.0 \\
$1 s_{5}$ & $4 s[3 / 2]_{2}$ & 11.55 \\
$1 s_{4}$ & $4 s[3 / 2]_{1}$ & 11.62 \\
$1 s_{3}$ & $4 s^{\prime}[1 / 2]_{0}$ & 11.72 \\
$1 s_{2}$ & $4 s^{\prime}[1 / 2]_{1}$ & 11.83 \\
$2 p_{10}$ & $4 p[1 / 2]_{1}$ & 12.91 \\
$2 p_{9}$ & $4 p[5 / 2]_{3}$ & 13.08 \\
$2 p_{8}$ & $4 p[5 / 2]_{2}$ & 13.09 \\
$2 p_{7}$ & $4 p[3 / 2]_{1}$ & 13.15 \\
$2 p_{6}$ & $4 p[3 / 2]_{2}$ & 13.17 \\
$2 p_{5}$ & $4 p[1 / 2]_{0}$ & 13.27 \\
$2 p_{4}$ & $4 p^{\prime}[3 / 2]_{1}$ & 13.28 \\
$2 p_{3}$ & $4 p^{\prime}[3 / 2]_{2}$ & 13.30 \\
$2 p_{2}$ & $4 p^{\prime}[1 / 2]_{1}$ & 13.33 \\
$2 p_{1}$ & $4 p^{\prime}[1 / 2]_{0}$ & 13.48 \\
\hline
\end{tabular}


Table 2 Paschen and Racah notation of state levels of Xe I [24]

\begin{tabular}{lll}
\hline Paschen notation & Xenon $(\mathrm{Xe} \mathrm{I})$ & \\
\cline { 2 - 3 } & Racah notation & Energy \\
\hline$g s$ & $5 p^{6}$ & 0 \\
$1 s_{5}$ & $6 s[3 / 2]_{2}$ & 8.31 \\
$1 s_{4}$ & $6 s[3 / 2]_{1}$ & 8.43 \\
$1 s_{3}$ & $6 s^{\prime}[1 / 2]_{0}$ & 9.44 \\
$1 s_{2}$ & $6 s^{\prime}[1 / 2]_{1}$ & 9.56 \\
$2 p_{10}$ & $6 p[1 / 2]_{1}$ & 9.58 \\
$2 p_{9}$ & $6 p[5 / 2]_{2}$ & 9.68 \\
$2 p_{8}$ & $6 p[5 / 2]_{3}$ & 9.72 \\
$2 p_{7}$ & $6 p[3 / 2]_{1}$ & 9.78 \\
$2 p_{6}$ & $6 p[3 / 2]_{2}$ & 9.82 \\
$2 p_{5}$ & $6 p[1 / 2]_{0}$ & 9.93 \\
$2 p_{4}$ & $6 p^{\prime}[3 / 2]_{1}$ & 10.95 \\
$2 p_{3}$ & $6 p^{\prime}[3 / 2]_{2}$ & 11.05 \\
$2 p_{2}$ & $6 p^{\prime}[1 / 2]_{1}$ & 11.06 \\
$2 p_{1}$ & $6 p^{\prime}[1 / 2]_{0}$ & 11.14 \\
\hline
\end{tabular}

reaction of Argon and Xenon have been selected from LxCat [28], an open access hub of database with several parameters needed for plasma modelling.

The calculation of the reaction rates has been performed under the hypothesis of local thermodynamic equilibrium, thus considering a Maxwellian electron energy distribution function with the following formula:

$K_{i j}=\sqrt{\frac{2 e}{m}} \int_{0}^{\infty} \varepsilon \sigma_{i j} f_{0} \mathrm{~d} \varepsilon$

Where $f_{0}$ is the electron energy distribution function expressed as:

$f_{0}(\varepsilon)=2\left(\frac{1}{T_{e}^{3} \pi}\right)^{1 / 2} \exp \left(\frac{-\varepsilon}{T_{e}}\right)$

in Eq. (7), $\sigma_{i j}$ is the electron-impact integrated cross section for transition from the lower state $i$ to the upper state $j, \varepsilon$ is the electron energy in $\mathrm{eV}, T_{e}$ is the electron temperature in $\mathrm{eV}$, and $\mathrm{m}$ is the electron mass. The inverse reaction rates have been computed using the principle of detailed balancing [6]. An analytical formula has been developed for the inverse cross section of the considered reaction and has been integrated using Eq. (7).

The following equation has been introduced considering a generic reaction, defined as follows:

$e+A \rightarrow e+B$

with $A$ and $B$ two generic states of a heavy species. From [6], the electron and the heavy species are related through the detailed balance principle (Eq. 9), and with the energy balance, (Eq. 10):

$m_{R}^{2} g_{A} v_{R}^{2} \sigma\left(v_{R}\right)=m_{R}^{* 2} g_{B} v_{R}^{* 2} \sigma^{*}\left(v_{R}^{*}\right)$

$\frac{1}{2} m_{R} v_{R}^{2}=\frac{1}{2} m_{R}^{*} v_{R}^{* 2}+e \Delta E_{i j}$

where $\sigma^{*}\left(v_{R}^{*}\right)$ is the inverse cross section for the reduced velocity of the system after the impact, $\Delta E_{i j}=E_{j}-E_{i}$ is the threshold energy, $g_{A}, g_{B}$ are the statistical weights of the initial and final excited species, and finally $m_{R}$ and $m_{R}^{*}$ are the reduced masses of the system before and after the collision, that in the case of electronic impact are equal. Assuming, $\varepsilon=\frac{1}{2} m_{R} v_{R}^{2} / e$, with e the electron charge, it is possible to rewrite Eqs. (9) and (10) as:

$g_{A} \varepsilon \sigma(\varepsilon)=g_{B} \varepsilon^{*} \sigma^{*}\left(\varepsilon^{*}\right)$

$\varepsilon=\varepsilon^{*}+e \cdot \varepsilon_{a}$

substituting Eq. (12) in Eq. (11):

$\sigma^{*}\left(\varepsilon-\Delta E_{i j}\right)=\frac{g_{A}}{g_{B}} \frac{\varepsilon}{\varepsilon-\Delta E_{i j}} \sigma(\varepsilon)$

The last formula has been implemented in the model to calculate the inverse rate coefficient.

\subsection{Radiative Reactions}

In the model, only spontaneous emissions are taken into account from the $2 p$ levels to $1 s$ levels and from resonant $1 s$ levels to ground state, for either Argon and Xenon. The reactions are listed in "Appendix". The radiative effect on the populating/depopulating mechanisms depends on, $A_{i j}$, the Einstein coefficient of the reaction and, $\Lambda_{i j}$, the escape factor. Einstein coefficients are coefficients of proportionality that express the measure of probability of the emission of photons by an atom. The escape factor instead is an adimensional parameter used to take into account reabsorption effects due to radiation trapping [29]. This last effect has been modelled through Mewe's formula [30], that for a uniform distribution of emitting and absorbing atoms is defined as:

$\Lambda_{i j}=\frac{2-e^{-\rho \kappa_{i j} / 1000}}{1+\rho \kappa_{i j}}$

where $\rho$ is the plasma characteristic length, and $\kappa_{i j}$ is the reabsorption coefficient for transition $\mathrm{i}$ to $\mathrm{j}$. In case of lowtemperature plasma, only Doppler broadening is significant [21] and the reabsorption coefficient can be expressed as: 
$\kappa_{i j}=\frac{g_{i}}{g_{j}} \frac{\lambda_{i j}^{3}}{8 \pi^{3 / 2}} n_{j} A_{i j} \sqrt{\frac{M}{2 R T_{g}}}$

where $\lambda_{i j}$ is the wavelength corresponding to the optical transition $i \rightarrow j, \mathrm{M}$ is the molar mass, $\mathrm{R}$ is the gas constant, and $T_{g}$ is the temperature of the neutral atoms, that in the model is assumed constant.

\subsection{Lumping Methodology}

To reduce the number of equations to be implemented inside fluid solvers, a lumping of the chemical reactions is usually performed. This process consists of reducing, by averaging, the number of reactions, diminishing in this way the computational cost of the simulation. The total number of species considered in the chemical model for both Argon and Xenon is sixteen: $G S, 1 s_{5}, \ldots, 1 s_{2}$ $, 2 p_{10}, \ldots, 2 p_{1}$, one ion species and electrons. To implement the selected reactions into 3D-VIRTUS, we have reduced the number of excited species into the six fictitious species presented in Table 3:

Different formulas have been developed for the lumping of the direct and inverse reaction rates for the collisional reaction, and for the escape factor of the radiative reactions. Under a hypothesis of local thermodynamic equilibrium and making use of the Boltzmann relation, we have obtained:

$K_{l}^{\mathrm{dir}}=\sum_{i}^{N_{i}}\left[\frac{\sum_{j}^{N_{j}} K_{i j}}{\sum_{k}^{N_{i}}\left(\frac{g_{k}}{g_{i}} \exp \left(-\frac{U_{k}-U i}{K_{B} T}\right)\right)}\right]$

where $i=1,2, \ldots N_{i}$ and $j=1,2, \ldots N_{j}$, and with $N_{i} \times N_{j}$ equal to the total number of reactions. The previous formula has been implemented to lump the direct reactions, such as $1 s_{M} / 1 s_{R} \rightarrow 2 P /$ ion and $2 P \rightarrow$ ion.

Regarding the inverse rate coefficient, the formula can be obtained from Eq. (16) considering the inverse reaction $K_{j i}$ and inverting the indices $\mathrm{j}$ and $\mathrm{i}$. Then, making use of the principle of detail balance, the formula can be written as function of the rate coefficient $K_{i j}$ :
$K_{l}^{\mathrm{inv}}=\sum_{j}^{N_{j}}\left[\frac{\sum_{i}^{N_{i}} K_{i j} g_{i} \exp \left(-\frac{U_{i}}{K_{B} T}\right)}{\sum_{k}^{N_{j}} g_{k} \exp \left(-\frac{U_{k}}{K_{B} T}\right)}\right]$

Moreover, the energy levels and the statistical weights have been lumped with the following criteria:

$E_{l}^{i j}=\frac{\sum_{i}^{N_{i}} \sum_{j}^{N_{j}}\left(g_{i} E_{i j}\right)}{\sum_{k}^{N_{i}} g_{k}}$

$G_{l}^{i}=\sum_{i}^{N_{i}}\left(g_{i}\right)$

For the radiative reactions: $1 s_{R} \rightarrow G S, 2 P \rightarrow 1 s_{M} / 1 s_{R}$, the lumping has been performed on the self-absorption factor with the following empirical formula.

$\kappa_{\text {lump }_{i j}}=\frac{\sum_{i} g_{i} \kappa_{i j}}{\sum_{i} g_{i}}$

where $\mathrm{i}$ are the starting levels, and $\mathrm{j}$ are the levels of arrival. The obtained average value has been inserted in Eq. (14) to obtain the lumped escape factor required for the spontaneous emission of the fictitious species.

\section{Numerical Simulations}

The developed CRM has been tested to verify the numerically integrated rate coefficients on an experimental Inductively Coupled Plasma (ICP) fed with Argon [20], performing a comparison against the rate coefficients obtained exploiting Bolsig + [27]. The comparison has been done between the distribution of $1 s$ and $2 p$ excited states population with the experimental measurements given in [20]. Then, to verify the lumping methodology, the electron density, $n_{e}$, and electron temperature, $T_{e}$, given by the detailed and lumped models have been compared. For the Xenon model, the calculated density distribution of the $1 s$ and $2 p$ excited states of an ICP has been compared to the results given by another CRM in literature [24]. Finally, the lumped Argon model was implemented in 3D-VIRTUS and the populations distribution of the lumped species is shown. The resulting electron density has been compared to experimental measurements.
Table 3 Table of lumped species

\begin{tabular}{ll}
\hline GS & Ground state \\
$1 s_{M}$ & $1 s_{5}, 1 s_{3}$ (metastable) \\
$1 s_{R}$ & $1 s_{4}, 1 s_{2}$ (resonant) \\
$2 \mathrm{P}$ & $2 p_{10}, 2 p_{9}, 2 p_{8}, 2 p_{7}, 2 p_{6}, 2 p_{5}, 2 p_{4}, 2 p_{3}, 2 p_{2}, 2 p_{1}$ \\
Ion & 1 st ionization \\
$\mathrm{e}$ & Electron \\
\hline
\end{tabular}


Table 4 Input parameter of Argon ICP simulation [20]

\begin{tabular}{ll}
\hline Pressure $(\mathrm{Pa})$ & 1 \\
Dimensions $(\mathrm{mm})$ & $400 \times 150 \times 150$ \\
Power $(\mathrm{W})$ & 75 \\
$T_{e}(\mathrm{eV})$ & 3 \\
$n_{e}\left(\mathrm{~m}^{-3}\right)$ & $3 \times 10^{16}$ \\
$T_{g}(\mathrm{~K})$ & 400 \\
\hline
\end{tabular}

Table 5 Datasets of input excitation cross sections of Argon model for ICP simulation

\begin{tabular}{llll}
\hline$g s \rightarrow 1 s$ & $g s \rightarrow 2 p$ & $1 s \rightarrow 1 s$ & $1 s \rightarrow 2 p$ \\
\hline BSR [31, 32] & BSR [31, 32] & BSR [31, 32] & BSR [31, 32] \\
\hline
\end{tabular}

\subsection{ICP Simulations}

\subsubsection{Argon Model}

The first validation of the Argon model has been preformed on an experimental case of an Inductively Coupled Plasma (ICP). The ICP experimental setup [20] consists of a quartz cylinder inserted in a stainless steel chamber fed with Argon gas. The cylinder is wrapped with an induction coil that, once coupled to the plasma column, provides the ionization energy. The electron density has been measured with a Langmuir probe located in the center of the plasma source, while the intensity of the optical emission of the discharge region has been recorded by a monochromator. The input parameters of the simulation are reported in Table 4 . The population densities of the excited levels have been computed using the Argon model and compared with the rate coefficients provided by Bolsig+. The cross sections required to calculate the rate coefficients have been selected from LxCat [28] and for the excitation reaction are listed in Table 5.

In Fig. 2, the two solutions have been compared against the experimental results in terms of population densities. On the left axis, the population density of the $2 p$ species, $n_{2 p}$, has been reported divided by its statistical weight, $g_{2 p}$. The values of $n_{2 p} / g_{2 p}$ have been normalized to one hundred. The $1 s$ levels are expressed in ppm, where $n_{1 s}, g_{1 s}, n_{g}$ are, respectively, the population density of $1 s$, and its degeneracy degree, and the neutral gas density. It can be noticed that the population distribution of the $1 s$ and $2 p$ levels given by the Maxwellian rate coefficients is generally more in agreement with the experimental results. The Maxwellian rate coefficients provide a better descritpion of the $2 p$ population distribution except for the $2 p_{6}$ level for which Bolsig+ gives a more accurate result. In particular, in comparison to the Maxwellian rate coefficients Bolsig+ returns
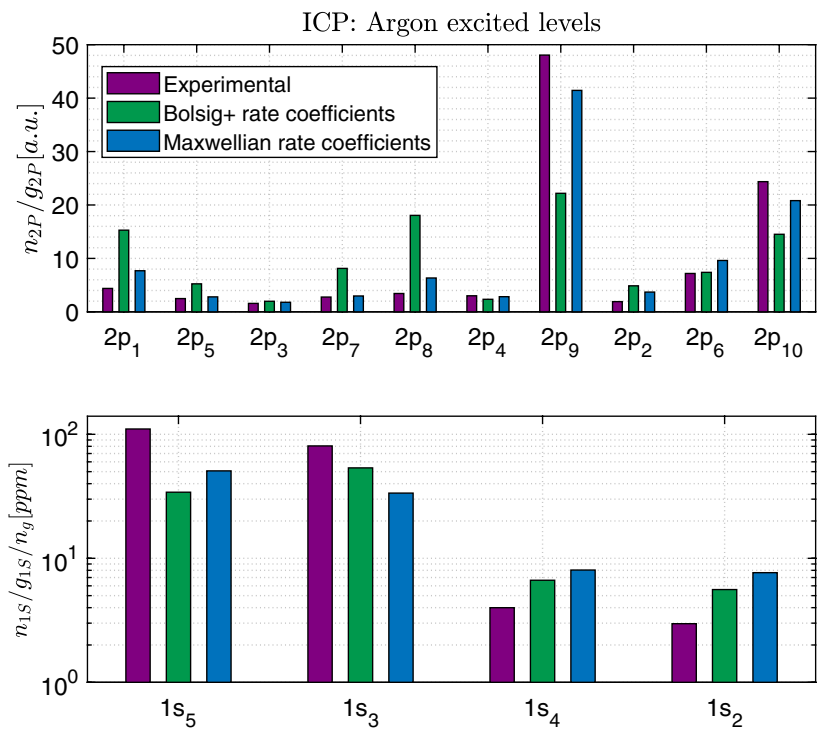

Fig. 2 Population density of Argon $1 s$ and $2 p$ levels from CRM using Maxwellian rate coefficients (blu bar) and using Bolsig+ (green bar) against the mean value of the OES measurements (violet bar) [20]. Here $n_{1 s}\left(n_{2 p}\right)$ refers to the population density of $1 s(2 p)$ levels, $n_{g}$ refers to the gas density and $g_{1 s}\left(g_{2 p}\right)$ is the degeneracy degree. The values of $n_{2 p} / g_{2 p}$ are normalized to one hundred
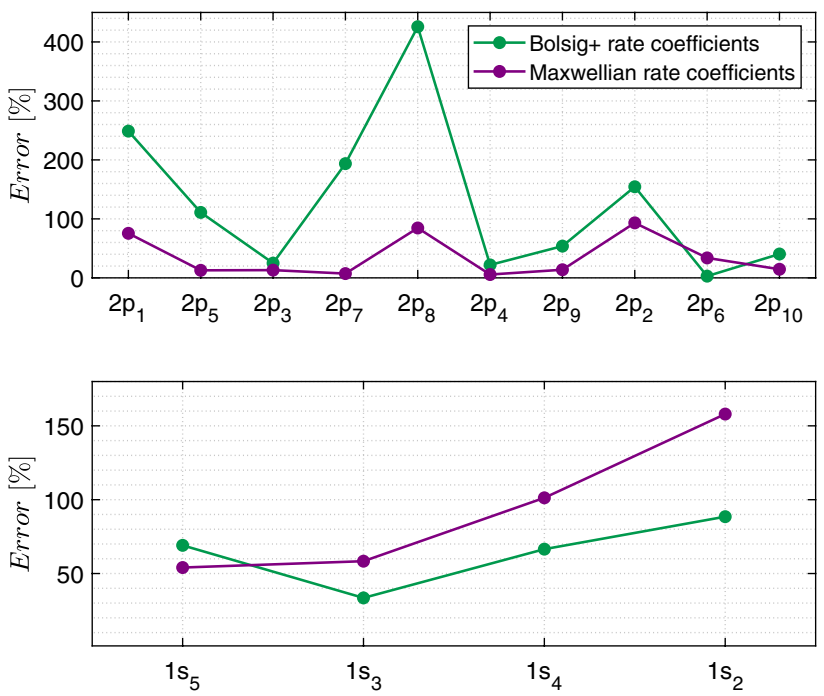

Fig. 3 Error of population distribution of excited levels of Argon, with respect to expected value of OES measurements [20]: using Maxwellian and Bolsig+ rate coefficients

significantly higher values for $2 p_{1}, 2 p_{5}, 2 p_{7}, 2 p_{8}, 2 p_{2}$ levels and lower values for $2 p_{9}, 2 p_{10}$. On the other hand, regarding the $1 s_{3}, 1 s_{4}, 1 s_{2}$ excited levels, Bolsig + represents better the population distribution, while for the $1 s_{5}$ the Maxwellian rate coefficient gives still a better result. In Fig. 3, the relative error of the two cases with respect to the mean value of the 
Table 6 Input parameter of Xenon ICP simulation [24]

\begin{tabular}{ll}
\hline Pressure $(\mathrm{Pa})$ & 61.3 \\
Radius and length $(\mathrm{mm})$ & $10 \times 290$ \\
Power $(\mathrm{W})$ & 45 \\
$T_{e}(\mathrm{eV})$ & 1.13 \\
$n_{e}\left(\mathrm{~m}^{-3}\right)$ & $9 \times 10^{17}$ \\
$T_{g}(\mathrm{~K})$ & 600 \\
\hline
\end{tabular}

Table 7 Input excitation cross sections of Xenon model

\begin{tabular}{llll}
\hline$g s \rightarrow 1 s$ & $g s \rightarrow 2 p$ & $1 s \rightarrow 1 s$ & $1 s \rightarrow 2 p$ \\
\hline BSR [31, 32] & BSR [31, 32] & Priti [24] & Priti [24] \\
\hline
\end{tabular}

experimental results is reported. The wide errors registered by the two models on the calculations of the density distribution of excited states are a characteristic common also to other reliable CRM present in literature [20, 24].

\subsubsection{Xenon Model}

For what concerns the model developed for Xenon, the results have been compared to those obtained in [24]. The input parameters of the simulation considered in this case are listed in Table 6.

The reference geometry of the ICP has been taken from [33]. The experimental setup consists of an ICP source with two concentric quartz tubes. The internal cylinder of length $29 \mathrm{~mm}$ and internal radius of $10 \mathrm{~mm}$, represents the plasma column. Whereas the external cylinder contains the induction coil, made of copper, and the refrigerating water. The database of cross sections for excitation reactions used for the following simulation is reported in Table 7, while the remaining reactions considered are listed in "Appendix". In Fig. 4, the obtained population distribution for the $1 \mathrm{~s}$ and $2 \mathrm{p}$ levels of the Xenon collisional radiative model is shown.

The population densities of $2 p 10-2 p 4$ levels show in general good agreement with the proposed model. On the other hand, the $2 p 3-2 p 1$ levels present an irregular behaviour; with all three points that exceed notably the values reported in Priti [24].The $1 s$ levels are generally lower in magnitude, especially the $1 s_{2}$ level that shows a difference of only $30 \%$ with respect to Priti's density.

The difference among the two models becomes clearer considering the deviation of the results with respect to Priti's model, presented in Fig. 5. Regarding the $1 s$ levels, the deviation is always below $100 \%$, similar to the results obtained for the Argon model. With, the $1 s_{2}$ level presents the smaller deviation from the results obtained by Priti. The deviation of the $2 p$ levels is below $50 \%$ for the first 6 levels, in order of increasing energy, except fot the $2 p_{6}$ level that presents a
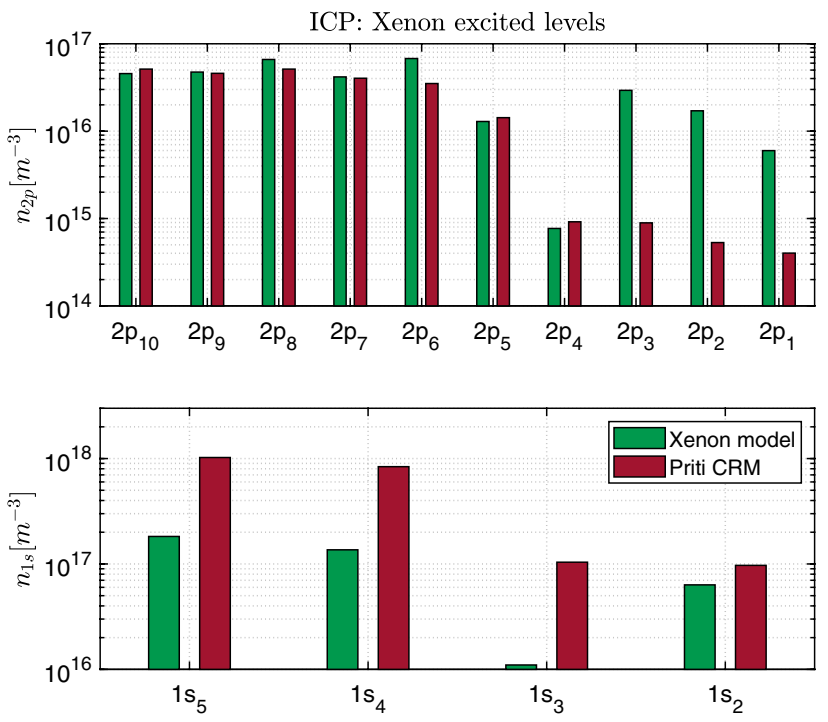

Fig. 4 Population density of Xenon $1 s$ and $2 p$ levels from CRM using Maxwellian rate coefficients (green bar) against Priti [24] CRM (red bar)
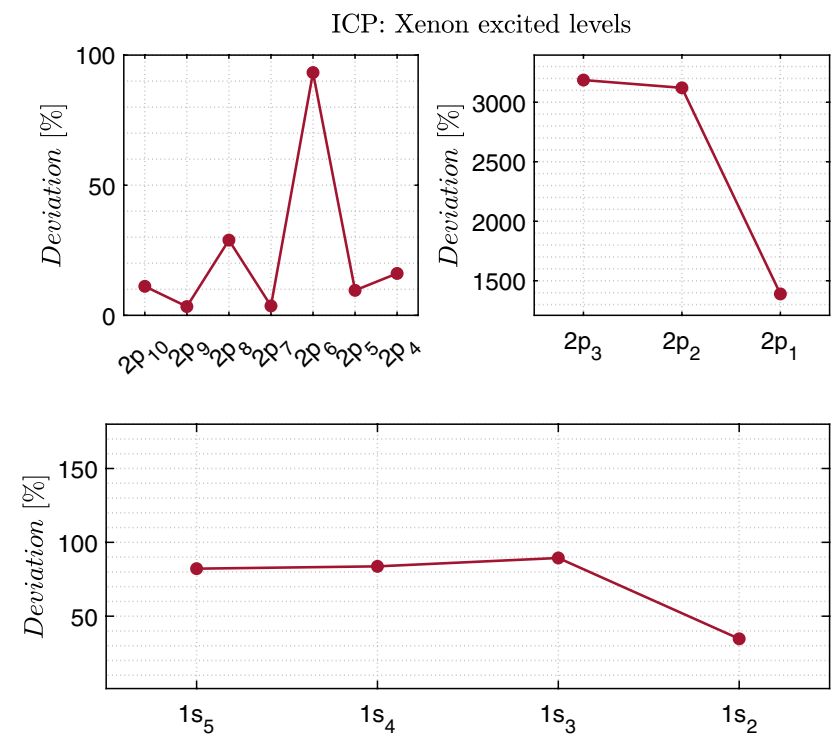

Fig. 5 Deviation of excited levels density distribution of Xenon model in comparison to Priti's model [24]

deviation around $100 \%$. The last three levels, $2 p_{3}, 2 p_{2}, 2 p_{1}$ present the highest deviation, suggesting the necessity to improve the modelling of the reactions that concern those levels.

Considering the wide deviation of calculated density distributions, the results obtained can be considered in agreement with those presented by Priti et al. for the $1 s_{5}, \ldots, 1 s_{2}$ and $2 p_{10}, \ldots, 2 p_{4}$ levels. On the contrary, the model needs an improvement for the reactions concerning the $2 p_{3}, \ldots 2 p_{1}$ levels. This result can be explained considering that with 
Table 8 Excitation input cross sections and analytical rate coefficients of Argon model implemented into 3D-VIRTUS

\begin{tabular}{llll}
\hline$g s \rightarrow 1 s$ & $g s \rightarrow 2 p$ & $1 s \rightarrow 1 s$ & $1 s \rightarrow 2 p$ \\
\hline an. rate [20] & an. rate [20] & BSR [31, 32] & BSR [31, 32] \\
\hline
\end{tabular}

respect to the Argon model, for the Xenon model there is less available data in literature, such as transitions parameters for radiative reactions and cross sections for $1 s_{3} \rightarrow 2 p$ transitions.

\subsection{D-VIRTUS}

The Argon model has been implemented into 3D-VIRTUS and tested for a typical plasma discharge inside an HPT. The obtained results have been compared to the experimental case reported in [34]. These experimental measurements were performed on a Piglet reactor filled with Argon gas, where the electron density has been measured with a Langmuir probe located in the center line of the Helicon discharge. To implement the chemical model's output into the fluid solver, the rate coefficients have been lumped with the aforementioned formulas. For the excited states reactions, the rate coefficients considered are a mix of analytical rates taken from literature [20] and rates integrated from the cross sections. The cross sections and analytical rate coefficients used for the de/excitation reactions are reported in Table 8.

The experimental setup consists of a $20 \mathrm{~cm}$ long Pyrex source tube connected to a $28.8 \mathrm{~cm}$ aluminium chamber. The source region is connected with a turbomolecular pump system that keeps the chamber's base pressure at $0.39 \mathrm{~Pa}$. The $\mathrm{RF}$ antenna, that deposits power into the gas, is located in the source region operating with a frequency of $13.56 \mathrm{MHz}$ and generating a power of $250 \mathrm{~W}$. The magnetic field can be generated through the source or exhaust coil. In the following validation, the magneto-static field has been considered generated by the exhaust coil with a maximum intensity of $21 \mathrm{G}$.

The experimental set-up considered is fully 3D since the antenna configuration is not axisymmetric. Nevertheless, it has been noticed [18] that the differences in the results assuming a 2D-axisymmetric problem are not significantly different from a case of a 3D domain. In the simulation, the antenna has been assumed radially distributed around the dielectric walls of the source chamber. The simulation considers a 2D-axisymmetric mesh of 11,000 nodes, as shown in Fig. 6. The species considered inside the plasma discharge are reported in Table 3 . The results regarding the population distribution of the excited levels are reported in Figs. 7, 8 and 9.

All three species are mainly distributed in a region around the antenna, with an increased density near the walls of the

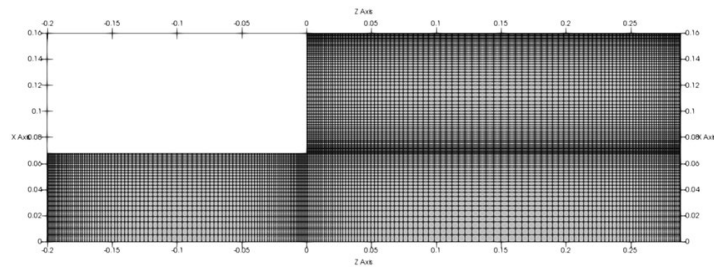

Fig. 6 Fluid mesh of the Piglet reactor

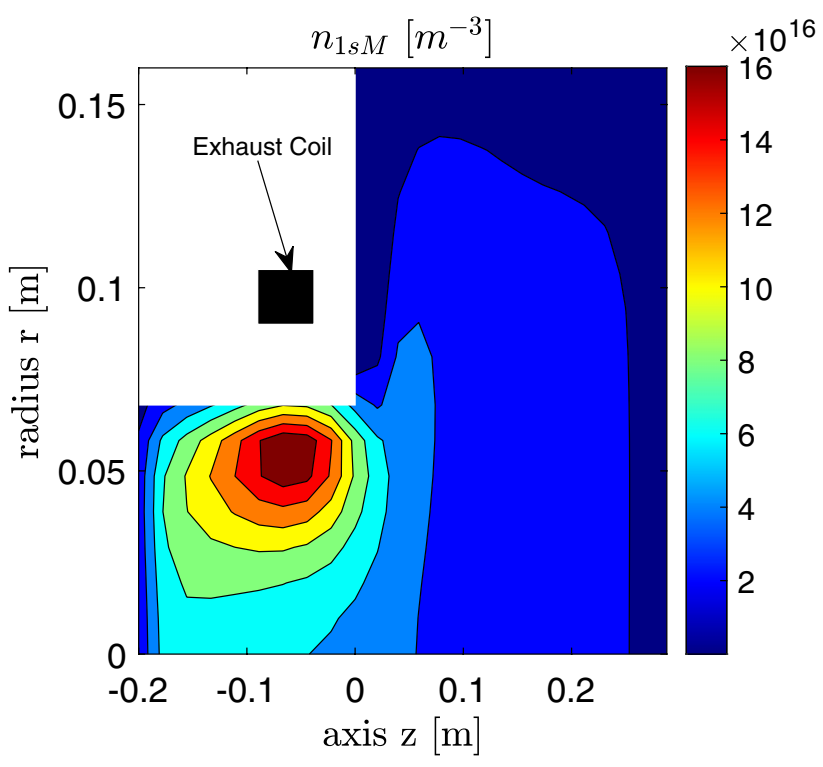

Fig. $71 s_{M}$ density distribution

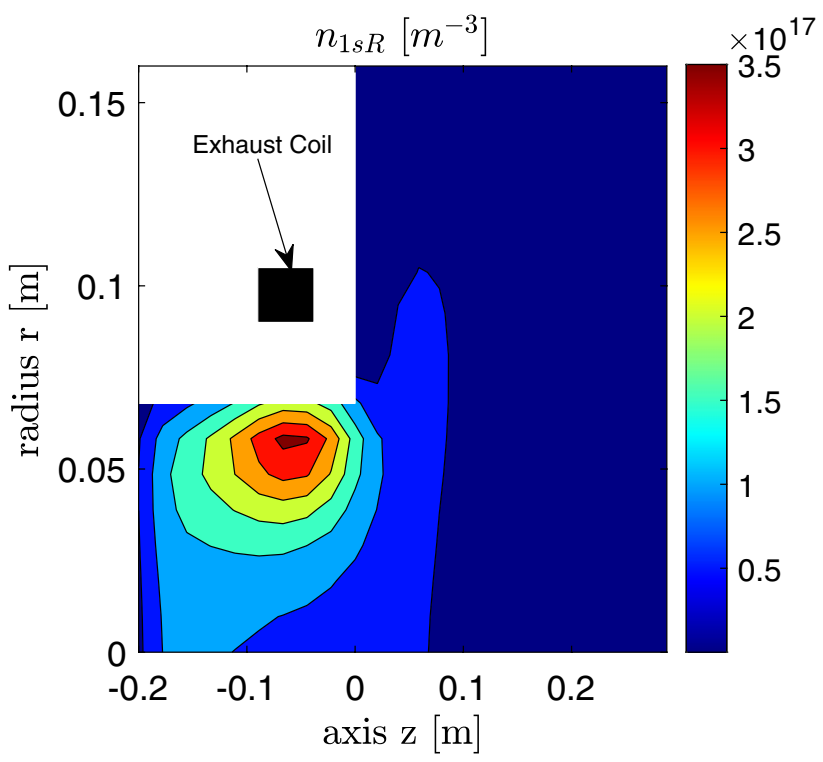

Fig. $81 s_{R}$ density distribution 


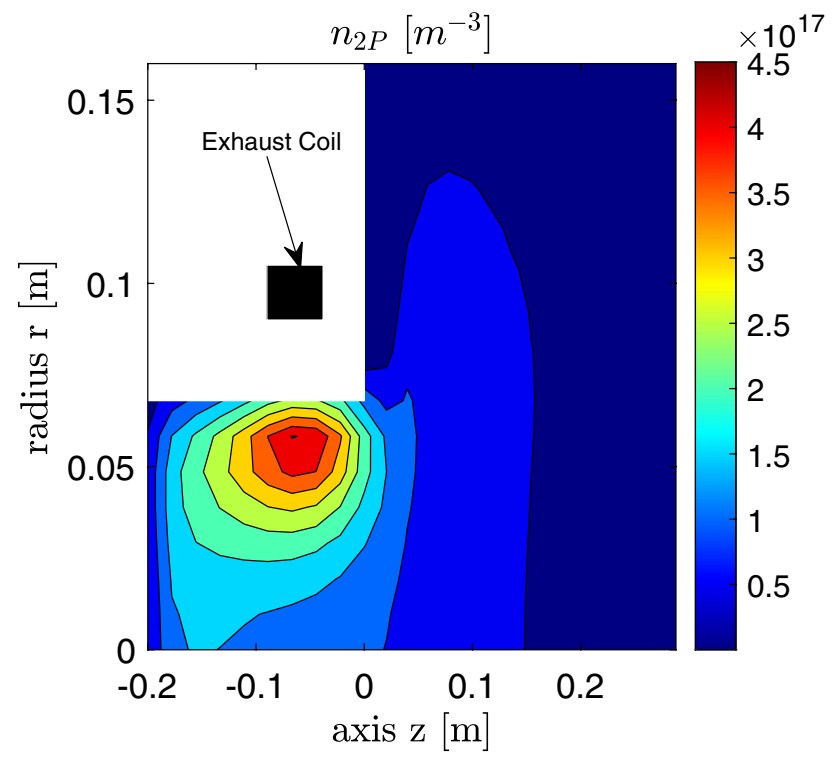

Fig. $92 P$ density distribution

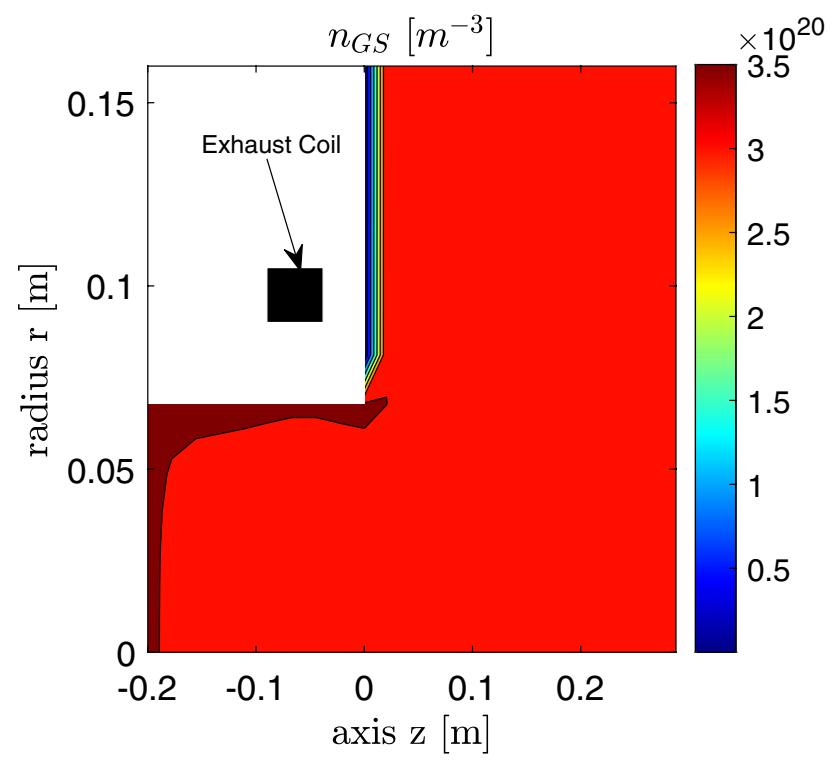

Fig. 10 Ground states density distribution

reactor. This is coherent with the working principle of the HPT since the ionization and excitation effects of neutral atoms are more relevant in regions next to the antenna's wall, where there is more deposited power and the electrons have higher kinetic energies. The Metastable $1 s$ levels have a lower density distribution but in a more spread area with respect to the $1 s$ Resonant levels. This is probably due to the shorter lifetime of the Resonant $1 s$ levels which tend to decay quickly through optical radiation. Also, $2 P$ levels are more concentrated in the region around the exhaust coil with a magnitude around $4 \times 10^{17} \mathrm{~m}^{-3}$, while are less present

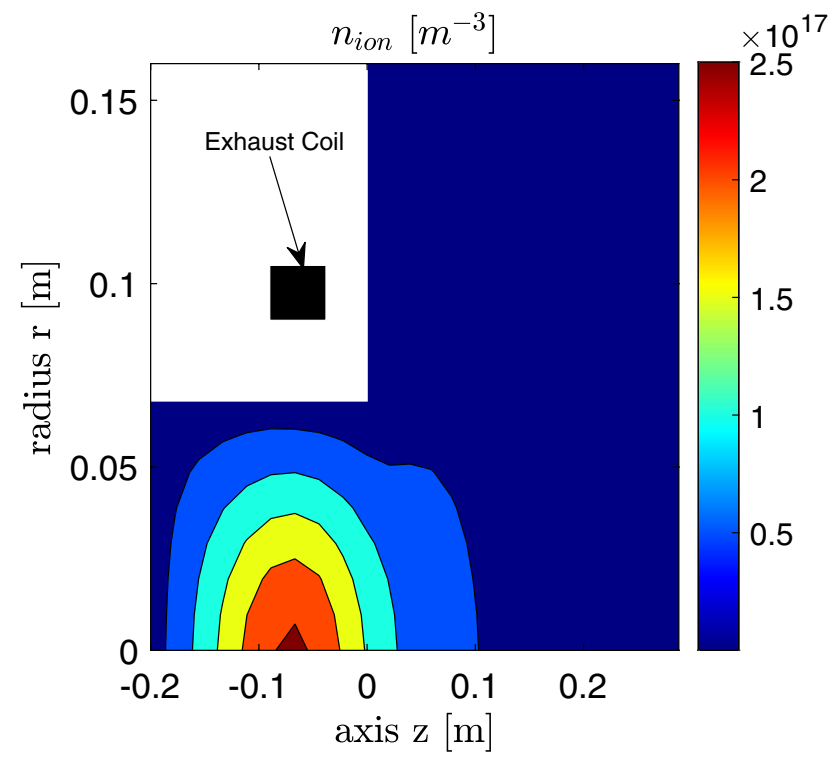

Fig. 11 Ion density distribution

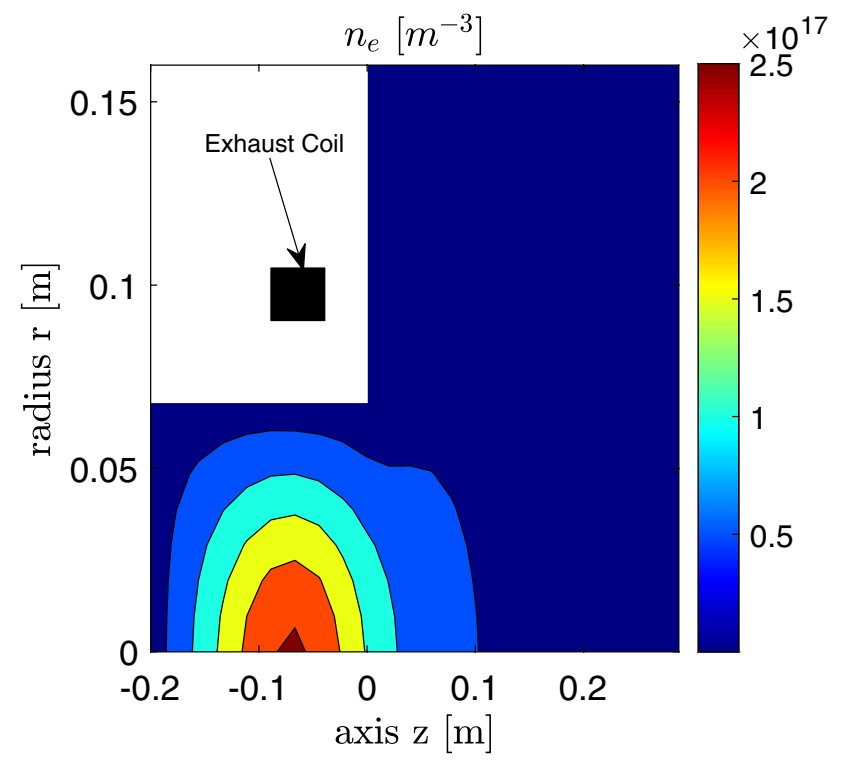

Fig. 12 Electron density distribution

in the diffusion chamber. The neutral density distribution reported in Fig. 10 shows that the density is uniformly distributed along the domain with a slight increase in proximity of the outlet.

For the ion and electron density, results are reported in Figs. 11 and 12. In this case, the density population of ions and electrons is almost equally distributed in space, enforcing the fundamental assumption of quasi-neutral plasma. Due to the recombination at the walls, the deposited power profile leads to a distribution of electrons and ions concentrated in the center of the source region. For what concerns 


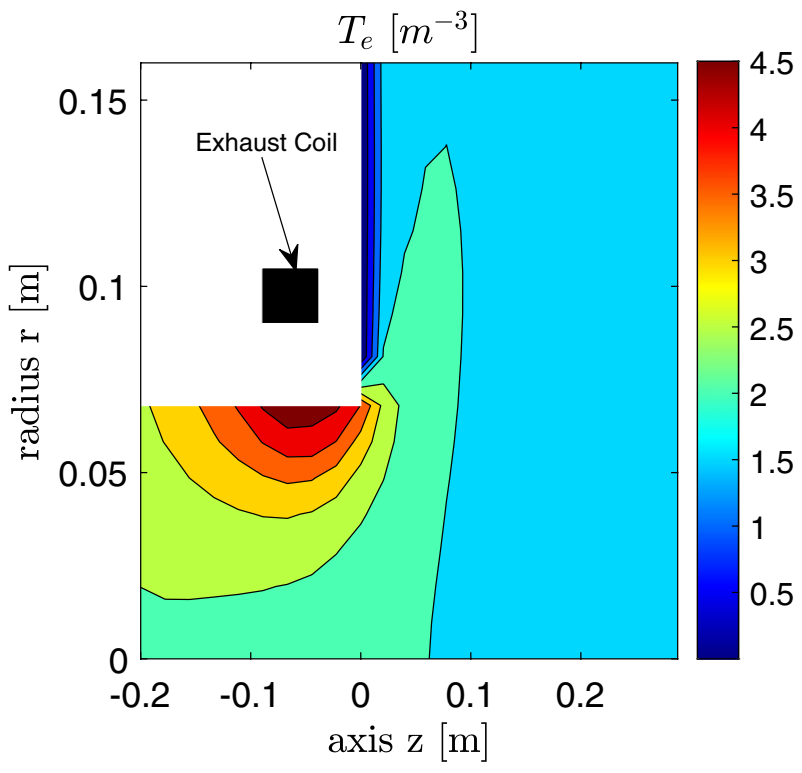

Fig. 13 Electron temperature distribution

the electron temperature, in Fig. 13, it can be observed how considerable is the spatial variation, coherently with the fact that electrons are the only species which is not under isothermal hypothesis. Thus, variations of up to $2 \mathrm{eV}$ are registered between the source chamber and the diffusion chamber (Fig. 14).

Finally, a comparison of the electron density with the experimental data reported in [34] is presented, including an uncertainty band evaluated by [18]. The latter has been added considering an uncertainty of the measure of \pm $20-30 \%$. The uncertainty has been evaluated considering

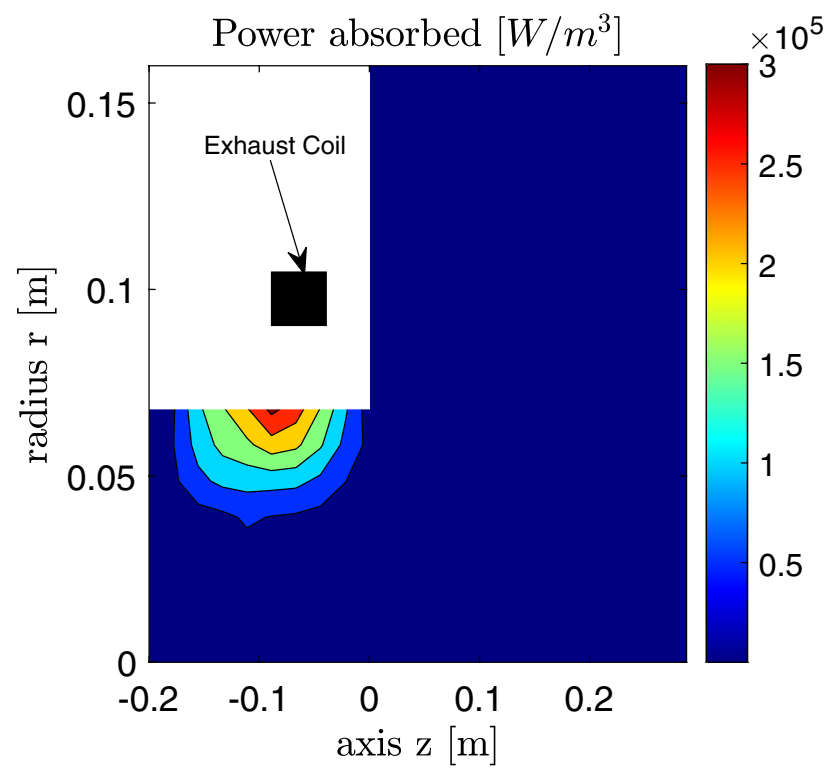

Fig. 14 Power density distribution errors that may arise by the perturbation of the probe on the system when it is inserted in the source region. The measurements have been performed with a Langmuir probe on the central axis of the Piglet reactor $(r=0 \mathrm{~m})$.

Figure 15 shows that 3D-VIRTUS with the developed CRM is able to track the experimental results inside the uncertainty band, being the difference in the middle region between the computed values and the measurements around $25 \%$.

For what concerns the computational effort, the simulation time is about $15 \mathrm{~min}$ on a quad-core Intel machine. So, the chemical model with the lumped procedure proved to be reliable and suited for multidimensional fluid codes.

\section{Predictive Performance Simulation}

In this section, a predictive analysis of the propulsive performances of an Helicon Plasma Thruster (HPT) is presented. The analysis has been performed through a Global Model, with implemented the propulsive parameters of a magnetic nozzle treated in [8]. The magnetic nozzle is produced at the outlet of the plasma source from the diverging lines of the magnetic field. Because of the low-pressure regime in which helicon thrusters operate, ions have been considered collisionless. Moreover, due to ionization and radial plasma losses, the plasma and neutral gas densities have been considered to vary axially in the source chamber. The thrust considered is generated essentially through two reaction mechanisms. The pressure of the gas, $\left(F_{0}\right)$, and the Lorentz force, $\left(F_{\mathrm{mag}}\right)$. In the first case, the force generated depends on the pressure of the gas and is exerted at the bottom of the source chamber. In the latter case, the force is created by the radial component of the

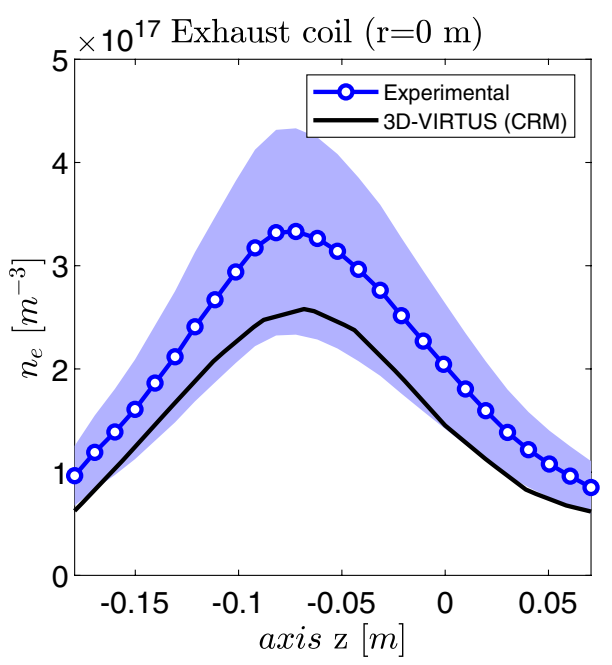

Fig. 15 Comparison of measured and calculated electron densities of a Piglet reactor on the central axis $(r=0 \mathrm{~m})$, for the Exhaust coil experimental case [34]. The blue area represents the expected uncertainty band 
divergent magnetic field and the azimuthal electron current induced by the magnetic field itself. Another contribution is given by the non ionized neutral gas, coming out from the discharge chamber. The thrust generated by the neutral particles is equal to:

$F_{\mathrm{gas}}=\dot{m} u_{e}+q n_{g} T_{g} A_{e}$

where $u_{e}$ is the exhaust velocity of the gas, $T_{g}$ is the gas temperature in $\mathrm{eV}, n_{g}$ is the gas density, and $A_{e}$ is the exit cross-sectional area. Given that the flow rate of neutral gas is $\dot{m}=m_{i} n_{g} v_{g} A$, by substituting $n_{g}$, derived from the latter, in the gas thrust equation, we obtain:

$F_{\text {gas }}=\dot{m} v_{g}\left(1+\frac{q T_{g}}{m_{i} v_{g}^{2}}\right)$

The force exerted by the plasma on the bottom of the thruster is obtained by multiplying the pressure by the cross-sectional area of the chamber:

$F_{0}=q \beta n_{0} T_{e} A_{0}$

where $A_{0}$ is the cross-sectional area of the source tube, $\beta$ is a parameter used to define the radial variation of the plasma density, $n_{0}$ is the axial plasma density at the bottom of the source chamber, $T_{e}$ is the electron temperature in $\mathrm{eV}$. Considering the momentum conservation equation applied next to the outlet:

$\frac{\mathrm{d}}{\mathrm{d} z}\left(m_{i} \beta n v_{z}^{2}\right)=q \beta n E_{z}$

it is possible to get the magnetic contribution of the thrust, which results from the action of the divergent magnetic field:

$F_{\text {mag }}=\int_{V} J_{\Theta} B_{r} \mathrm{~d} V$

with $J_{\theta}$ the azimuthal current resulting from the application of the divergent magnetic field and $B_{r}$ the radial component of the magnetic field. In conclusion, the total thrust can be ontained as the total contribution of the three forces:

$F_{\text {tot }}=F_{0}+F_{\text {mag }}+F_{\text {gas }}$

The performance parameters in the model, the specific impulse, $I_{s p}$, and the thrust efficiency, $\eta_{T}$, have been computed with the following formulas:

$I_{\mathrm{sp}}=\frac{F_{\mathrm{tot}}}{\dot{m} g_{0}}, \quad \eta_{T}=\frac{F_{\mathrm{tot}}^{2}}{2 \dot{m} P_{T}}$

with $g_{0}$ being the gravitational acceleration at sea level and $P_{\text {tot }}$ the total power absorbed by the plasma divided for the efficiency of the power transfer.
Table 9 Input parameters of Global Model for the propulsive performance analysis

\begin{tabular}{lll}
\hline N.test & $\begin{array}{l}\text { Mass flow } \\
\text { rate }(\mathrm{mg} / \mathrm{s})\end{array}$ & $\begin{array}{l}\text { Deposited } \\
\text { power } \\
\text { (W) }\end{array}$ \\
\hline 01 & 0.20 & 38 \\
02 & 0.20 & 59 \\
03 & 0.20 & 122 \\
04 & 0.17 & 39 \\
05 & 0.17 & 59 \\
06 & 0.17 & 97 \\
\hline
\end{tabular}
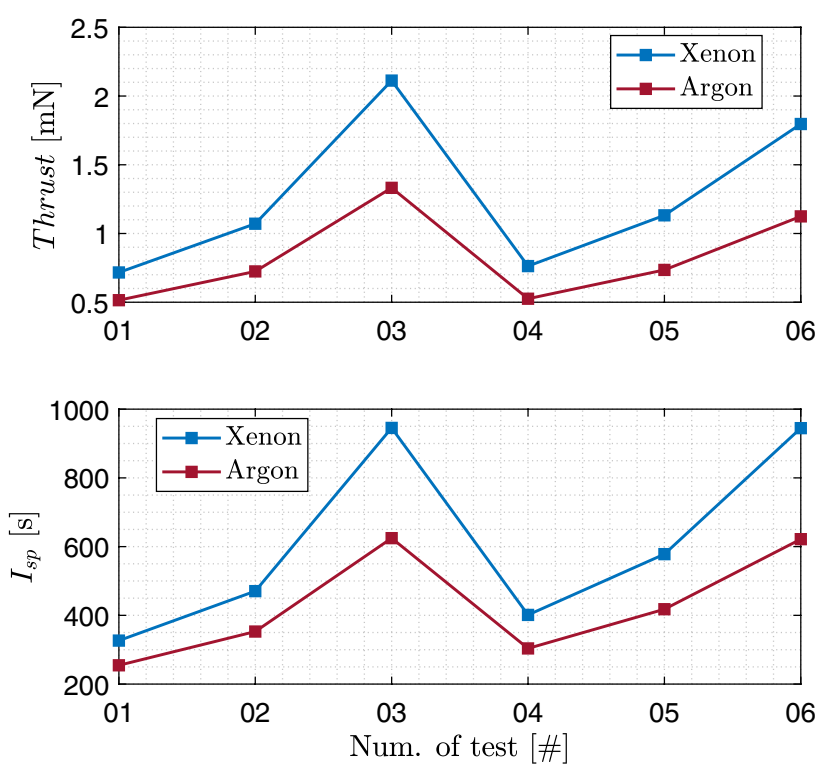

Fig. 16 Thrust and specific impulse $\left(I_{\mathrm{sp}}\right)$ obtained for each test case, given by Xenon and Argon model

For what concern the chemical model, for Xenon, the CRM has been set up with the excitation cross sections reported in Table 7, while for Argon the input cross sections for the excited states are those of Table 8 . The input mass flow and deposited power for the simulations are reported in Table 9, for both Argon and Xenon.

The values obtained for thrust and specific impulse are in accordance with the general results obtained for discharges of this type [8]. In particular, from Fig. 16, the values of thrust for Xenon oscillate in a range from 0.7 to $2.1 \mathrm{mN}$, while for the specific impulse, a maximum value of $950 \mathrm{~s}$ is reached. On the other hand, the Argon model registers lower performance than Xenon, with thrust ranging from 0.5 to $1.3 \mathrm{mN}$ and a maximum specific impulse of $600 \mathrm{~s}$. In Fig. 17, thrust is compared with the delivered power for the two inflows of propellant gases.

The figure shows that diminishing the inflow mass the output thrust increases, with a stronger tendency for higher 


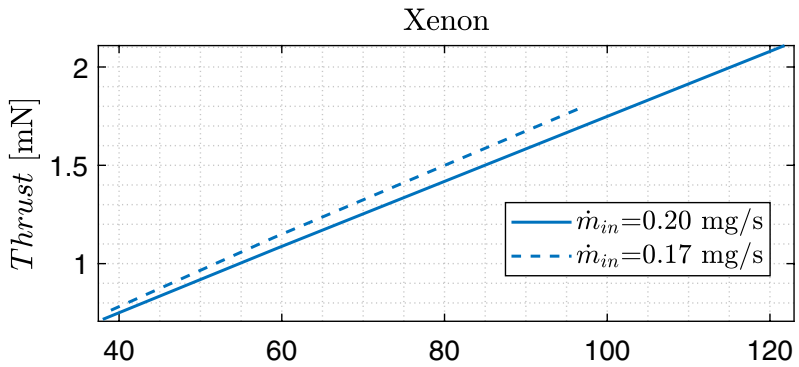

Argon

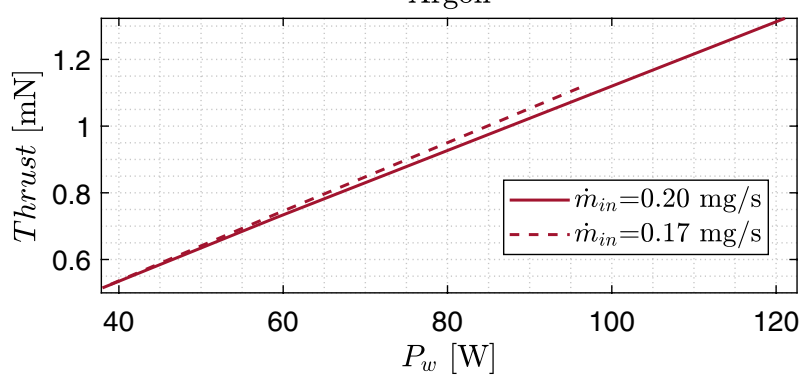

Fig. 17 Thrust versus power $\left(P_{w}\right)$ for different mass flows, given by Xenon and Argon model

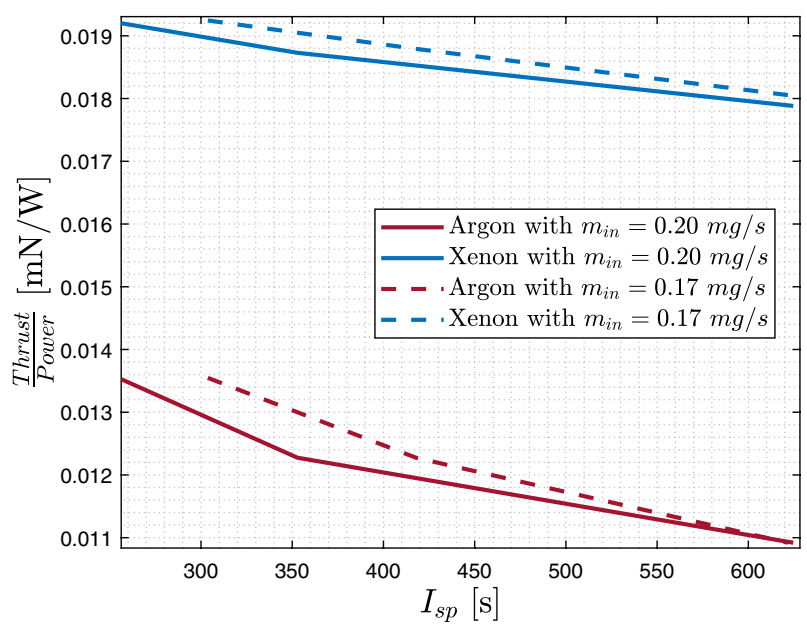

Fig. 18 Thrust-to-power ratio versus specific impulse $\left(I_{\mathrm{sp}}\right)$, given by Xenon and Argon model

power values. To better compare the propulsive results of Xenon and Argon, the ratio of thrust over power with respect to the specific impulse is presented in Fig. 18. It can be observed that reducing the inflow the thrust to power ratio increases for both the propellants. Nevertheless, the specific impulse increases for lower values of thrust to power ratio for both Argon and Xenon. In all cases, Xenon displays almost two times better values with respect to Argon.

The estimated efficiency of the two systems is shown in Fig. 19. As expected, Xenon displays better results than

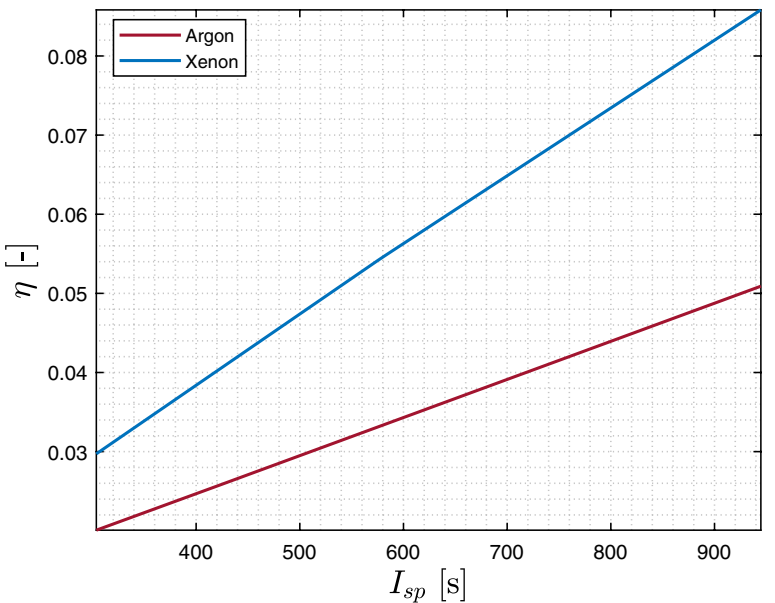

Fig. 19 Efficiency versus specific impulse $\left(I_{\mathrm{sp}}\right)$, for Xenon and Argon model

Argon, thanks to its higher atomic mass and lower ionization energy, with values ranging from 3 to $9 \%$. Argon instead for the considered cases reaches a maximum efficiency of $5 \%$. The low values of efficiency are typical of HPT [5] due to their acceleration mechanism. However, a low efficiency can be tolerated since in common space applications, the electric power required by electric thrusters is produced limitlessly by solar panels. So, more emphasis shall be given to the specific impulse, which represents the efficiency of the propellant used and presents an optimal value for the given mass of the power plant system.

In conclusion, it can be stated that the Xenon-fed HPT performs better than the Argon one, and this is generally coherent with the theoretical considerations on electric thrusters, as shown also in [35].

\section{Conclusion}

In this work, a simple collisional radiative model has been presented for Argon and Xenon plasma discharges. The model includes bulk reactions, with a focus to the $1 s$ and $2 p$ excited states. The rate coefficients of the considered reactions have been exploited from cross sections, but when available, some analytical reaction rates have also been used. Moreover, a lumping procedure to reduce the number of reactions has been presented allowing the implementation into the fluid module of 3D-VIRTUS.

The first validation of the Argon model has shown good agreement with the experimental results of an ICP discharge. The second validation has been done implementing the model into 3D-VIRTUS, which has shown that the computed electron density falls within the band of uncertainty of the experimental measurements. A verification of the Xenon model has been 
done comparing the population's density of excited states with the results of another CRM, but an accurate validation shall be performed in a future work. Finally, a predictive analysis of HPT performances for both Xenon and Argon was presented, showing reasonable results for both propellants. In a future investigation, a test campaign shall be performed on a HPT using a dedicated thrust stand to validate the obtained results. Further studies can take into consideration the implementation of excitation and de-excitation reactions among the higher excited states of the atom to extend the validity of the CRM in a wider range of applications.

\section{Appendix: Detailed Reactions of Argon and Xenon Model}

See Tables 10, 11, 12, 13 and 14.

Table 10 Table of electron impact ionization and scattering reactions: analytical rate and input cross section

\begin{tabular}{lll}
\hline & Argon model & Xenon model \\
\hline Ionization & & \\
$G S+e \rightleftharpoons$ ion $+e$ & {$[31,32]$} & {$[36]$} \\
$1 s+e \rightleftharpoons$ ion $+e$ & an. rate [20] & {$[37]$} \\
$2 p+e \rightleftharpoons$ ion $+e$ & an. rate [20] & {$[37]$} \\
Elastic scattering & & \\
$G S+e \rightleftharpoons G S+e$ & an. rate [20] & {$[36]$} \\
$1 s+e \rightleftharpoons 1 s+e$ & an. rate [20] & {$[36]$} \\
$2 p+e \rightleftharpoons 2 p+e$ & an. rate [20] & {$[36]$} \\
\hline
\end{tabular}

Funding Open access funding provided by Alma Mater Studiorum Università di Bologna within the CRUI-CARE Agreement.

Table 11 Excitation and de-excitation reactions implemented in the Argon model

\begin{tabular}{lll}
\hline GS de/excitation & 1 s de/excitation & 2 p de/excitation \\
\hline$G S+e \rightleftharpoons 1 s_{5}+e$ & $1 s_{5}+e \rightleftharpoons 1 s_{4}+e$ & $1 s_{3}+e \rightleftharpoons 2 p_{4}+e$ \\
$G S+e \rightleftharpoons 1 s_{4}+e$ & $1 s_{5}+e \rightleftharpoons 1 s_{2}+e$ & $1 s_{3}+e \rightleftharpoons 2 p_{2}+e$ \\
$G S+e \rightleftharpoons 1 s_{3}+e$ & $1 s_{3}+e \rightleftharpoons 1 s_{4}+e$ & $1 s_{2}+e \rightleftharpoons 2 p_{8}+e$ \\
$G S+e \rightleftharpoons 1 s_{2}+e$ & $1 s_{3}+e \rightleftharpoons 1 s_{2}+e$ & $1 s_{2}+e \rightleftharpoons 2 p_{7}+e$ \\
$G S+e \rightleftharpoons 2 p_{10}+e$ & $1 s_{5}+e \rightleftharpoons 2 p_{10}+e$ & $1 s_{2}+e \rightleftharpoons 2 p_{6}+e$ \\
$G S+e \rightleftharpoons 2 p_{9}+e$ & $1 s_{5}+e \rightleftharpoons 2 p_{9}+e$ & $1 s_{2}+e \rightleftharpoons 2 p_{3}+e$ \\
$G S+e \rightleftharpoons 2 p_{8}+e$ & $1 s_{5}+e \rightleftharpoons 2 p_{8}+e$ & $1 s_{2}+e \rightleftharpoons 2 p_{2}+e$ \\
$G S+e \rightleftharpoons 2 p_{7}+e$ & $1 s_{5}+e \rightleftharpoons 2 p_{7}+e$ & $1 s_{2}+e \rightleftharpoons 2 p_{1}+e$ \\
$G S+e \rightleftharpoons 2 p_{6}+e$ & $1 s_{5}+e \rightleftharpoons 2 p_{6}+e$ & $1 s_{4}+e \rightleftharpoons 2 p_{10}+e$ \\
$G S+e \rightleftharpoons 2 p_{5}+e$ & $1 s_{5}+e \rightleftharpoons 2 p_{4}+e$ & $1 s 4_{+} e \rightleftharpoons 2 p_{7}+e$ \\
$G S+e \rightleftharpoons 2 p_{4}+e$ & $1 s_{5}+e \rightleftharpoons 2 p_{3}+e$ & $1 s 4_{+} e \rightleftharpoons 2 p_{6}+e$ \\
$G S+e \rightleftharpoons 2 p_{3}+e$ & $1 s_{5}+e \rightleftharpoons 2 p_{2}+e$ & $1 s 4_{+} e \rightleftharpoons 2 p_{5}+e$ \\
$G S+e \rightleftharpoons 2 p_{2}+e$ & $1 s_{3}+e \rightleftharpoons 2 p_{10}+e$ & $1 s 4_{+} e \rightleftharpoons 2 p_{3}+e$ \\
$G S+e \rightleftharpoons 2 p_{1}+e$ & $1 s_{3}+e \rightleftharpoons 2 p_{7}+e$ & $1 s 4_{+} e \rightleftharpoons 2 p_{2}+e$ \\
\hline
\end{tabular}

Table 12 Excitation and de-excitation reactions implemented in the Xenon model

\begin{tabular}{lll}
\hline GS de/excitation & 1 s de/excitation & 2 p de/excitation \\
\hline$G S+e \rightleftharpoons 1 s_{5}+e$ & $1 s_{5}+e \rightleftharpoons 1 s_{4}+e$ & $1 s_{2}+e \rightleftharpoons 2 p_{5}+e$ \\
$G S+e \rightleftharpoons 1 s_{4}+e$ & $1 s_{5}+e \rightleftharpoons 1 s_{2}+e$ & $1 s_{2}+e \rightleftharpoons 2 p_{4}+e$ \\
$G S+e \rightleftharpoons 1 s_{3}+e$ & $1 s_{3}+e \rightleftharpoons 1 s_{4}+e$ & $1 s_{2}+e \rightleftharpoons 2 p_{3}+e$ \\
$G S+e \rightleftharpoons 1 s_{2}+e$ & $1 s_{3}+e \rightleftharpoons 1 s_{2}+e$ & $1 s_{2}+e \rightleftharpoons 2 p_{2}+e$ \\
$G S+e \rightleftharpoons 2 p_{10}+e$ & $1 s_{5}+e \rightleftharpoons 2 p_{10}+e$ & $1 s_{2}+e \rightleftharpoons 2 p_{1}+e$ \\
$G S+e \rightleftharpoons 2 p_{9}+e$ & $1 s_{5}+e \rightleftharpoons 2 p_{9}+e$ & $1 s_{4}+e \rightleftharpoons 2 p_{10}+e$ \\
$G S+e \rightleftharpoons 2 p_{8}+e$ & $1 s_{5}+e \rightleftharpoons 2 p_{8}+e$ & $1 s_{4}+e \rightleftharpoons 2 p_{9}+e$ \\
$G S+e \rightleftharpoons 2 p_{7}+e$ & $1 s_{5}+e \rightleftharpoons 2 p_{7}+e$ & $1 s_{4}+e \rightleftharpoons 2 p_{7}+e$ \\
$G S+e \rightleftharpoons 2 p_{6}+e$ & $1 s_{5}+e \rightleftharpoons 2 p_{6}+e$ & $1 s_{4}+e \rightleftharpoons 2 p_{6}+e$ \\
$G S+e \rightleftharpoons 2 p_{5}+e$ & $1 s_{5}+e \rightleftharpoons 2 p_{5}+e$ & $1 s_{4}+e \rightleftharpoons 2 p_{5}+e$ \\
$G S+e \rightleftharpoons 2 p_{4}+e$ & $1 s_{2}+e \rightleftharpoons 2 p_{10}+e$ & $1 s_{4}+e \rightleftharpoons 2 p_{4}+e$ \\
$G S+e \rightleftharpoons 2 p_{3}+e$ & $1 s_{2}+e \rightleftharpoons 2 p_{9}+e$ & $1 s_{4}+e \rightleftharpoons 2 p_{3}+e$ \\
$G S+e \rightleftharpoons 2 p_{2}+e$ & $1 s_{2}+e \rightleftharpoons 2 p_{7}+e$ & $1 s_{4}+e \rightleftharpoons 2 p_{2}+e$ \\
$G S+e \rightleftharpoons 2 p_{1}+e$ & $1 s_{2}+e \rightleftharpoons 2 p_{6}+e$ & $1 s_{4}+e \rightleftharpoons 2 p_{1}+e$ \\
\hline
\end{tabular}

Table 13 Radiative reactions implemented in the Argon model

\begin{tabular}{lll}
\hline Radiative decay & & \\
\hline $1 s_{4} \rightarrow G S+\hbar \omega$ & $2 p_{10} \rightarrow 1 s_{3}+\hbar \omega$ & $2 p_{3} \rightarrow 1 s_{4}+\hbar \omega$ \\
$1 s_{2} \rightarrow G S+\hbar \omega$ & $2 p_{7} \rightarrow 1 s_{3}+\hbar \omega$ & $2 p_{2} \rightarrow 1 s_{4}+\hbar \omega$ \\
$2 p_{10} \rightarrow 1 s_{5}+\hbar \omega$ & $2 p_{4} \rightarrow 1 s_{3}+\hbar \omega$ & $2 p_{8} \rightarrow 1 s_{2}+\hbar \omega$ \\
$2 p_{9} \rightarrow 1 s_{5}+\hbar \omega$ & $2 p_{2} \rightarrow 1 s_{3}+\hbar \omega$ & $2 p_{7} \rightarrow 1 s_{2}+\hbar \omega$ \\
$2 p_{8} \rightarrow 1 s_{5}+\hbar \omega$ & $2 p_{10} \rightarrow 1 s_{4}+\hbar \omega$ & $2 p_{6} \rightarrow 1 s_{2}+\hbar \omega$ \\
$2 p_{7} \rightarrow 1 s_{5}+\hbar \omega$ & $2 p_{8} \rightarrow 1 s_{4}+\hbar \omega$ & $2 p_{4} \rightarrow 1 s_{2}+\hbar \omega$ \\
$2 p_{6} \rightarrow 1 s_{5}+\hbar \omega$ & $2 p_{7} \rightarrow 1 s_{4}+\hbar \omega$ & $2 p_{3} \rightarrow 1 s_{2}+\hbar \omega$ \\
$2 p_{3} \rightarrow 1 s_{5}+\hbar \omega$ & $2 p_{6} \rightarrow 1 s_{4}+\hbar \omega$ & $2 p_{2} \rightarrow 1 s_{2}+\hbar \omega$ \\
$2 p_{2} \rightarrow 1 s_{5}+\hbar \omega$ & $2 p_{5} \rightarrow 1 s_{4}+\hbar \omega$ & $2 p_{1} \rightarrow 1 s_{2}+\hbar \omega$ \\
\hline
\end{tabular}

Table 14 Radiative reactions implemented in the Xenon model

Radiative decay

\begin{tabular}{ll}
\hline $1 s_{4} \rightarrow G S+\hbar \omega$ & $2 p_{10} \rightarrow 1 s_{4}+\hbar \omega$ \\
$1 s_{2} \rightarrow G S+\hbar \omega$ & $2 p_{7} \rightarrow 1 s_{4}+\hbar \omega$ \\
$2 p_{10} \rightarrow 1 s_{5}+\hbar \omega$ & $2 p_{6} \rightarrow 1 s_{4}+\hbar \omega$ \\
$2 p_{9} \rightarrow 1 s_{5}+\hbar \omega$ & $2 p_{5} \rightarrow 1 s_{4}+\hbar \omega$ \\
$2 p_{8} \rightarrow 1 s_{5}+\hbar \omega$ & $2 p_{3} \rightarrow 1 s_{2}+\hbar \omega$ \\
$2 p_{7} \rightarrow 1 s_{5}+\hbar \omega$ & $2 p_{4} \rightarrow 1 s_{2}+\hbar \omega$ \\
$2 p_{6} \rightarrow 1 s_{5}+\hbar \omega$ & $2 p_{2} \rightarrow 1 s_{2}+\hbar \omega$ \\
$2 p_{4} \rightarrow 1 s_{3}+\hbar \omega$ & $2 p_{1} \rightarrow 1 s_{2}+\hbar \omega$ \\
$2 p_{2} \rightarrow 1 s_{3}+\hbar \omega$ & \\
\hline
\end{tabular}

\section{Declarations}

Conflict of interest The authors have no conflict of interest to declare. 
Open Access This article is licensed under a Creative Commons Attribution 4.0 International License, which permits use, sharing, adaptation, distribution and reproduction in any medium or format, as long as you give appropriate credit to the original author(s) and the source, provide a link to the Creative Commons licence, and indicate if changes were made. The images or other third party material in this article are included in the article's Creative Commons licence, unless indicated otherwise in a credit line to the material. If material is not included in the article's Creative Commons licence and your intended use is not permitted by statutory regulation or exceeds the permitted use, you will need to obtain permission directly from the copyright holder. To view a copy of this licence, visit http://creativecommons.org/licenses/by/4.0/.

\section{References}

1. Goebel, D.M.: Fundamentals of Electric Propulsion, Ion and Hall Thrusters, 1st edn. Wiley, New York (2008). (ISBN: 978-0-470-42927-3)

2. Sutton, G.P., Biblarz, O.: Rocket Propulsion Elements, 8th edn. Wiley, New York (2010). (ISBN: 978-0-470-08024-5)

3. Wang, Y., et al.: A xenon collisional-radiative model applicable to electric propulsion devices: I. Calculations of electron-impact cross sections for xenon ions by the Dirac B-spline R-matrix method. Plasma Sources Sci. Technol. 28(10), 105004 (2019)

4. Pavarin, D., et al.: Plasma hydrazine: combined micro project overview and developmentstatus. In: Proceedings of the Space Propulsion Conference (2010)

5. Chen, F.F.: Helicon discharges and sources: a review. Plasma Sources Sci. Technol. 24(1), 014001 (2015)

6. Lieberman, M., Lichtenberg, J.: Principles of Plasma Discharges and Materials Processing. Wiley, New York (2005). (ISBN: 0-471-72001-1)

7. Takahashi, K: Helicon-type radiofrequency plasma thrusters and magnetic plasma nozzles. Rev. Mod. Plasma Phys. 3, 20 (2019)

8. Eur, T.L.: Helicon plasma thruster discharge model. Phys. Plasmas 21, 4 (2014)

9. Eduardo, A., Mario, M.: On plasma detachment in propulsive magnetic nozzles. Phys. Plasmas 18, 5 (2011)

10. Squire, J.P., et al.: Progress in experimental research of the Vasimr engine. Fusion Sci. Technol. 43(1T), 111-117 (2003)

11. Navarro-Cavallé, J., et al.: Experimental characterization of a 1 kW helicon plasma thruster. Vacuum 149, 69-73 (2018). ((ISSN: 0042- 207X))

12. Manente, M., et al.: REGULUS: a propulsion platform to boost small satellite missions. Acta Astron. 157, 241-249 (2019). ((ISSN: 0094- 5765))

13. Manente, M., et al.: REGULUS: iodine fed plasma propulsion system for small satellites (2019)

14. T4i-Technology for propulsion and innovation. https://www.t4inn ovation.com

15. Centro di Ateneo di Studi e Atività Spaziali 'Giuseppe Colombo' CISAS. https://cisas.unipd.it/

16. Bellomo, N., et al.: Enhancement of microsatellites' mission capabilities: integration of REGU- LUS electric propulsion module into UniSat-7. In: vol. 70th International Astronautical Congress, p. 52699 (2019)

17. Manente, M., Bellomo, N., Magarotto, M., et al.: Design and Inorbit demonstration of REGULUS, an iodine electric propulsion system. CEAS Sp. J. 20, 20 (2021)

18. Magarotto, M., Melazzi, D., Pavarin, D.: 3D- VIRTUS: equilibrium condition solver of radio-frequency magnetized plasma discharges for space applications. Comput. Phys. Commun. 247, 106953 (2020). ((ISSN: 0010-4655))

19. Melazzi, D., Lancellotti, V.: ADAMANT: a surface and volume integral-equation solver for the analysis and design of helicon plasma sources. Comput. Phys. Commun. 185(7), 1914-1925 (2014). ((ISSN: 0010-4655))

20. Zhu, X.-M., Pu, Y.-K.: A simple collisional-radiative model for low-temperature argon discharges with pressure ranging from $1 \mathrm{~Pa}$ to atmospheric pressure: kinetics of Paschen $1 \mathrm{~s}$ and $2 \mathrm{p}$ levels. J. Phys. D Appl. Phys. 43(1), 015204 (2009)

21. Evdokimov, K.E., et al.: Study of argon ions density and electron temperature and density in magnetron plasma by optical emission spectroscopy and collisional-radiative model. Resour. Efficient Technol. 3(2), 187-193 (2017). ((ISSN: 2405-6537))

22. Gangwar, R.K., Sharma, L., Srivastava, R.: Argon plasma modeling with detailed fine-structure cross sections. J. Appl. Phys. 20, 1 (2012)

23. Zhu, X-M., Yi-Kang, P.: A simple collisional-radiative model for low-pressure argon discharges. J. Phys. D Appl. Phys. 40(8), 2533-2538 (2007)

24. Gangwar, R.K., Srivastava, R.: Collisional-radiative model of xenon plasma with calculated electron impact fine structure excitation cross-sections. Plasma Sources Sci. Technol. 28(2), 025003 (2019)

25. NIST. Atomic Spectra Database. https://www.nist.gov/pml/ atomic-spectra-database

26. Nikolić, M., et al.: Measurements of population densities of metastable and resonant levels of argon using laser induced uorescence. J. Appl. Phys. 117, 023304 (2015)

27. Bolsig+. Electron Boltzmann Equation Solver. http://www.bolsig. laplace.univ-tlse.fr/

28. LXCAT. The Plasma Data Exchange Project. https://nl.lxcat.net/ home/

29. Boffard, J.B., et al.: Measurement of metastable and resonance level densities in rare gas plasmas by optical emission spectroscopy. Plasma Sources Sci. Technol. 18(3), 035017 (2009)

30. Mewe, R.: Relative intensity of helium spectral lines as a function of electron temperature and density. Br. J. Appl. Phys. 18(1), 107-118 (1967)

31. Zatsarinny, O., Bartschat, K.: B-spline Breit-Pauli R-matrix calculations for electron collisions with argon atoms. J. Phys. B Atom. Mol. Opt. Phys. 37(23), 4693-4706 (2004)

32. Allan, M., Zatsarinny, O., Bartschat, K.: Near-threshold absolute angle-differential cross sections for electron-impact excitation of argon and xenon. Phys. Rev. A 74,(2006)

33. Czerwiec, T., Graves, D.B.: Mode transitions in low pressure rare gas cylindrical ICP discharge studied by optical emission spectroscopy. J. Phys. D Appl. Phys. 37(20), 2827-2840 (2004)

34. Eur, T La., Charles, C., Boswell, R W. : Characterization of a helicon plasma source in low diverging magnetic fields. J. Phys. D Appl. Phys. 44(5), 055202 (2011)

35. Shabshelowitz, A.Gallimore, A.D., Peterson, P.Y.: Performance of a helicon hall thruster operating with xenon, argon, and nitrogen. J. Propuls. Power 30(3), 664-671 (2014)

36. Biagi, S.F.: Biagi V.8.97 Database. http://www.lxcat.net/Biagi

37. Hyman, H.A.: Electron-impact ionization cross sections for excited states of the rare gases ( $\mathrm{Ne}, \mathrm{Ar}, \mathrm{Kr}, \mathrm{Xe})$, cadmium, and mercury. Phys. Rev. A 20, 855-859 (1979)

Publisher's Note Springer Nature remains neutral with regard to jurisdictional claims in published maps and institutional affiliations. 